\title{
A colecção do Laboratorio Chimico
}

\author{
da Escola Politécnica de Lisboa. Novas perspectivas
}

PATRÍCIA POM B M E DEIROS*

A colecção do antigo Laboratorio Chimico da Escola Politécnica, hoje armazenada nas reservas do Museu de Ciência da Universidade de Lisboa (MCUL), goza, em termos museológicos, de características únicas. Neste texto, pretende-se brevemente explicar as razões de tal singularidade, mostrando, ao mesmo tempo, os desafios, oportunidades e responsabilidades que esta circunstância nos coloca.
O espaço físico originário da colecção, também hoje património do MCUL, é um admirável Laboratório Químico do século XIX - o antigo Laboratorio Chimico da Escola Polytechnica. Juntamente com o Amphiteatro anexo, representa, muito provavelmente, um dos únicos exemplares que restam dos grandes laboratórios químicos de ensino e de investigação oitocentistas na Europa. $\mathrm{Na}$ verdade, ao contrário do que aconteceu noutros países, que foram progressivamente modernizando os seus laboratórios e equipamentos, este Laboratorio permaneceu quase sempre em funcionamento, sem alterações espaciais significativas, até aos anos noventa do século XX, primeiro no contexto da Escola Politécnica (1837-1911), depois no contexto da Faculdade de Ciências da Universidade de Lisboa (1911- c.1998). ${ }^{1}$ Apesar do intenso uso que sofreu ao longo de mais de 150 anos, a sua traça manteve-se inalterada e ainda hoje possui grande parte do equipamento e mobiliário originais ${ }^{2}$ (hotes, forno, bancadas, armários, quadro de lousa, etc.).

O Laboratorio e o Amphiteatro funcionaram em pleno até 1998 mas, desde 2003, têm sido objecto de uma importante intervenção de conservação e restauro tendo em vista a sua integração

\footnotetext{
* Bolseira de Doutoramento em História da Ciência da Fundação para a Ciência e a Tecnologia. Museu de Ciência da Universidade de Lisboa, Rua da Escola Politécnica, 56, 1250-102 Lisboa (patpombo@yahoo.com)
}

museológica. ${ }^{3}$ O objectivo é apresentá-los ao público na sua configuração oitocentista conjuntamente com uma parte da colecção de Química correspondente ao seu funcionamento na época.

É esta colecção que, em grande medida, se encontra por estudar, quer em termos históricos, quer museológicos. Ela é constituída por um conjunto de objectos - reagentes, frascos mostruário com produtos naturais, colecções de minerais, material de laboratório em vidro, instrumentos científicos, modelos, painéis didácticos, entre outros - característicos do ensino e da investigação química no século XIX naquele espaço. Grande parte destes objectos foram recolhidos dos arrumos e áreas envolventes ao Laboratorio aquando da sua integração no MCUL. Deve realçar-se que, contrariamente às ciências naturais (botânica, zoologia, geologia, etc.), as práticas da física e química não determinam a constituição de colecções no sentido museológico do termo, sendo os objectos utilizados e re-utilizados em aparatos experimentais de ensino ou de investigação e depois progressivamente postos de lado, muitos deitados fora, à medida que se vão tornando ineficazes e obsoletos. No caso do Laboratorio Chimico, uma parte considerável dos objectos foi remetida ao longo de décadas para a cave do Anfiteatro, sótãos do edifício, armários e gabinetes de antigos professores. Aquando da integração do Laboratorio no MCUL, esses objectos foram incorporados pelo seu valor patrimonial e documental. É neste preciso momento da incorporação que o conjunto de objectos se torna numa colecção strictu sensu (Figuras de 1 a 5)

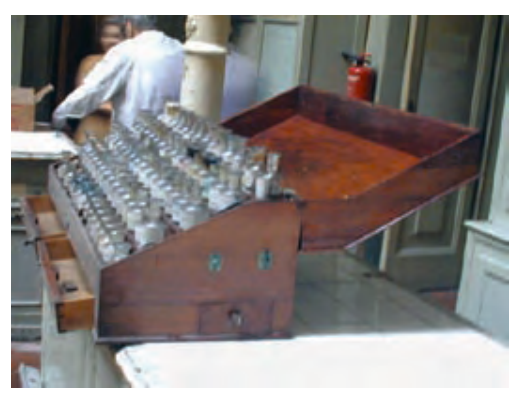

Figura 1 Piano de reagentes, recolhido em 2001 aquando dos trabalhos preparatórios para a recuperação do Laboratorio, número de inventário MCUL 1186 (foto: Marta Lourenço, cortesia MCUL)

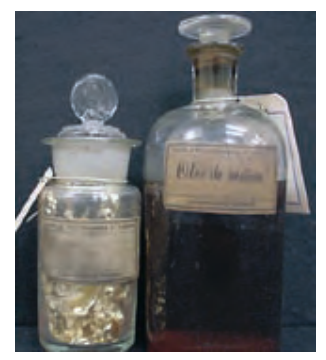

Figura 2 Frascos com a mesma tipologia de rótulo e com inscrição 'ESCOLA POLYTECHNICA 6. ' CADEIRA / LABORATORIO CHIMICO' contendo folha de ouro e óleo de resina (respectivamente números de inventário MCUL 1836 e MCUL 1842) (foto: Patrícia Medeiros, cortesia MCUL) 
Além do espaço de proveniência da colecção ser raro, autêntico e com elevados atributos estéticos, que foram, de resto, reconhecidas na própria época, ${ }^{4}$ um outro aspecto que amplia significativamente o seu valor é a existência, no arquivo do Museu, de um importante acervo gráfico que o documenta. Assim, antigos inventários, facturas, correspondência, fotografias, catálogos de fabricantes, registos de laboratório, ofícios, relatórios, manuais da época, entre muitos outros documentos, testemunham a vida do Laboratorio desde a fundação da Escola Politécnica, conferindo unicidade aos objectos da colecção e contribuindo, assim, para a determinação do seu significado histórico, científico e museológico (Figuras 6 e 7).

É este conjunto de aspectos que estabelecem um quadro histórico e museológico único para a colecção do Laboratorio Chimico, cuja singularidade importa aprofundar. Estão reunidos um conjunto de circunstâncias que criam um "terreno" museológico extremamente fértil e interessante para o seu estudo. Tomando como referência as "rupturas" de função e significado que a colecção sofreu quando passou do Laboratorio para a reserva do Museu e quando, num futuro breve, passar da reserva do Museu novamente para o Laboratorio musealizado, podemos considerar três estádios fundamentais na vida desta colecção: 1) a colecção no contexto de uso no Laboratorio ; 2) a colecção na reserva do MCUL; 3) a colecção em (futuro) contexto expositivo, de regresso ao Laboratorio, entretanto restaurado. Para cada um desses três estádios importa compreender a evolução dos significados que o conjunto colecção-espaçocontextos adquiriu, isto é, estudar a colecção e os objectos que a compõem na sua dupla articulação com o espaço e com os contextos (incluindo usos e pessoas) em que esteve, ou está, integrada. Significa isto que importa traçar o "percurso" histórico e museológico desta colecção procurando, em particular, compreender: 1) de que modo a colecção existente no antigo Laboratorio se articulava com as práticas de investigação e ensino aí desenvolvidas e com as concepções da Química da época, 2) de que modo esta colecção perdeu
Figura 3 Funil, número de inventário MCUL 2410 (foto: M. C. Elvas, cortesia MCUL). Repare-se na inscrição no vidro - '6. '. Cad'.

- relativa ao nome dado à disciplina de Química - a 6. ${ }^{a}$ Cadeira - desde a fundação da Escola Politécnica

Figura 4 Forno de incineração, número de inventário MCUL 3425 (foto: M. C. Elvas, cortesia MCUL)

Figura 5 Aparelho de Carré, número de inventário MCUL 2035 (foto: Sara Carvalho, cortesia MCUL)
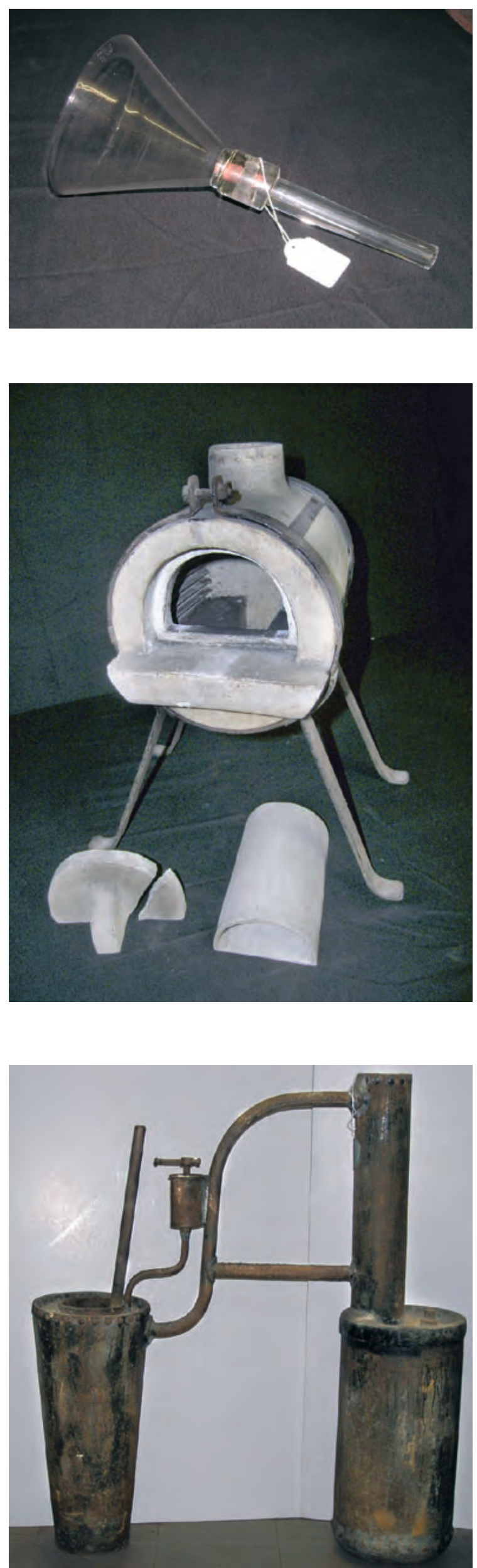


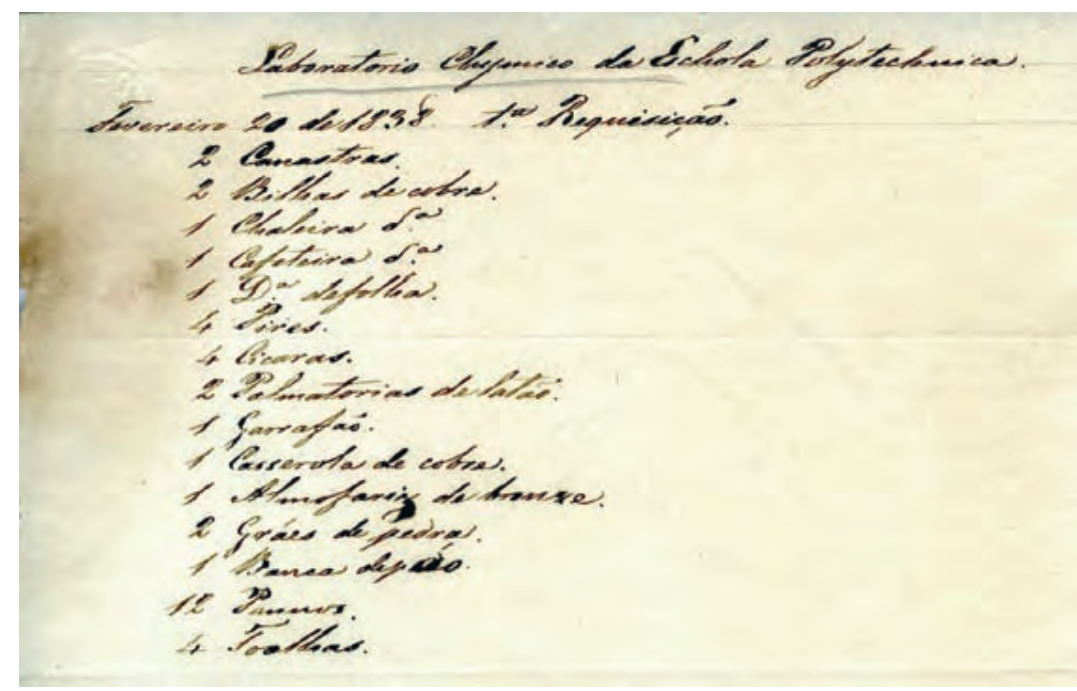

Figura 6 '1. ' Requisição' feita para o 'Laboratorio Chymico da Escola Polytechnica' datada de 20 de Fevereiro de 1838 (Digitalização: Filipe Ferreira, cortesia do Serviço de Biblioteca e Documentação, MCUL) a sua função original, foi descontextualizada, e integrada pelo museu (que procedimentos de incorporação, documentação, inventariação, classificação, conservação e estudo foram adoptados), 3) de que modo esta colecção (ou parte dela) vai ser recontextualizada e de novo reintegrada no Laboratorio $^{5}$, tendo em conta que essa reintegração será feita não exactamente no Laboratorio original, mas num espaço que, entretanto, também sofreu ruptura de função e descontextualização por via do restauro e musealização em curso. Qual o papel do

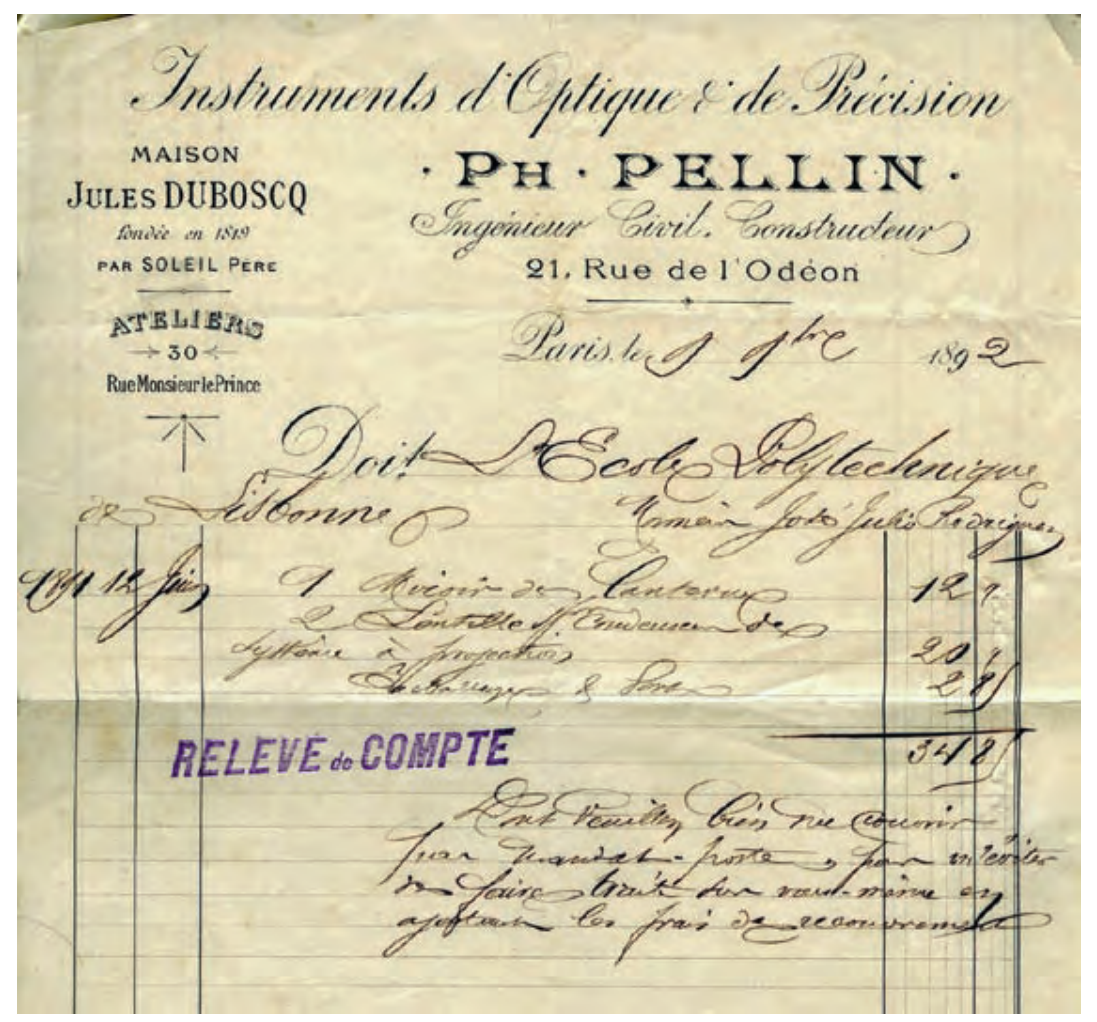

Figura 7 Factura de material dirigida ao cuidado do lente José Júlio Rodrigues, em 1892 (Digitalização: Filipe Ferreira, cortesia do Serviço de Biblioteca e Documentação, MCUL)
Laboratorio e da sua colecção na compreensão pública da ciência e imagens que veicula em termos de comunicação científica?

O significado desta colecção e dos objectos que a compõem não resulta apenas da sua materialidade ou qualidades intrínsecas como pode acontecer, por exemplo, no caso das obras de arte. Ao contrário destas, cuja singularidade é incontornável, o significado dos objectos desta colecção é em grande parte determinado pela sua trajectória, pela sua "biografia", como refere Alberti (2005) Sem ela, o seu significado será apenas ilustrativo ou arquetípico, o que traz limitações, por exemplo, a nível da interpretação pública destes objectos e da investigação. Fazer uma biografia desta colecção, aplicando as metodologias da cultura material que conferem uma atenção especial aos objectos e à gramática informativa da colecção (na linha de investigação que se designa usualmente como collection-based research), constitui, por isso, um desafio.

Para cada um dos estádios fundamentais da vida desta colecção, será necessário estudar a rede de relações que os seus objectos mantinham ou mantêm entre si, que mantinham ou mantêm com outros objectos, com o espaço, com as pessoas e com as práticas envolvidas. Em cada um desses momentos, dever-se-á procurar determinar a especificidade dessas relações. Assim, por exemplo, em contexto de uso no Laboratorio, os objectos mantinham relações dinâmicas sobretudo associados à funcionalidade (por exemplo, práticas de manipulação, aparatos experimentais) e estabeleciam relações com os utilizadores iniciais do Laboratorio (Lentes de Química, preparadores, alunos). Na reserva do MCUL, estabelecem relações com o pessoal do Museu (conservadores, investigadores, arquivistas, etc.) e encontram-se sobretudo reorganizados em função de critérios patrimoniais e de preservação material. Por último, em contexto expositivo, eles vão ser recontextualizados tendo por critério dominante o seu significado histórico, o qual será interpretado e comunicado a diferentes públicos (geral, escolar e especializado). 
Para além dos seus atributos imanentes (materiais de que são feitos, propriedades de natureza físico-química, características formais, etc.), estes objectos merecem ser interrogados nas suas múltiplas camadas de significação, nos diferentes níveis de informação que transportam consigo, questionados nas relações espacio-temporais que estabelecem entre si, investigados na sua cartografia relacional, olhados nas interferências directas e imediatas que mantêm com diversas esferas de fenómenos.

As mãos por onde passaram, a especificidade do saber-fazer neles envolvido, os traços e os sinais de uso, os estigmas funcionais que carregam consigo, os papéis que desempenharam, os lugares que habitaram e um sem número de outras particularidades relacionais, espaciais e de uso, constituem outras tantas marcas da sua "vida". Elas estão aí para serem lidas como instâncias concretas de memória, integradas numa "biografia", interpretadas no seu significado museológico, histórico e científico e descobertas, portanto, enquanto testemunhos relevantes para a Museologia, a História das Colecções e a História da Ciência, em particular para a História da Química em Portugal, tanto a nível de investigação como de ensino.

Notas

1 Em 22 de Abril de 1843, o funcionamento do Laboratório foi abruptamente interrompido pelo grande incêndio que destruiu quase por completo o antigo edifício do Colégio dos Nobres, local onde havia sido criada a Escola Polytechnica de Lisboa. O ensino da Química foi então transferido para a Casa da Moeda de Lisboa. De acordo com documentação existente no arquivo do MCUL, pensa-se que só em 1854 o Laboratório da Escola Politécnica voltou a funcionar, após as obras de (re)construção do edifício.

2 Refiro-me ao Laboratório já em finais do século XIX. Na verdade, entre 1888 e 1891, o Laboratorio Chimico sofre uma importante obra de reforma orientada pelo lente José Júlio Bettencourt Rodrigues (1845-1893). O laboratório é então dotado, entre outras coisas, de electricidade, água e gás canalizados, novos armários e bancadas de trabalho. Sobre este assunto consultar RAMALHO (2001).

3 Sobre o projecto de restauro e integração museológica consultar o recente artigo de LEITÃO \& RAMALHO (2006), publicado no número anterior da revista QUÍMICA, bem como o trabalho de RAMALHO (2001) que, em grande medida serviu de base ás obras de restauro efectuadas.

4 A qualidade funcional e estética atingida pelo laboratório nessa altura é então reconhecida pelo eminente químico August W. Hofmann (1818-1892) - que o classifica como um dos melhores estabelecimentos científicos da Europa - após a sua visita ao laboratório em carta dirigida a Júlio Rodrigues de 17 de Agosto de 1890. Ver RAMALHO (2001).

5 Outro aspecto interessante a estudar será a especificidade desta dupla-descontextualização relativamente a outros tipos museológicos, em que se verificam estes dois movimentos (caso, por exemplo, das casas-museu, fábricas musealizadas e dos sítios arqueológicos 'in situ')

6 Em termos metodológicos, a linha mestra desta investigação deverá ser construída com base nos estudos de cultura material, em especial nos trabalhos fundacionais de KOPYTOFF (1986), LUBAR \& KINGERY (1993) e KINGERY (1996), centrados na utilização de objectos como fonte primária de in- vestigação histórica. Estes estudos foram recentemente desenvolvidos por DASTON (2000, 2004) e ALBERTI (2005), que exploraram o conceito de 'biografia' aplicado a instrumentos científicos e a colecções, respectivamente.

\section{Bibliografia}

[1]. Caixa 26, "Cadeiras 6. ${ }^{a}, 7^{a}$ e Secção Mineralogica", Serviço de Biblioteca e Documentação, Museu de Ciência da Universidade de Lisboa.

[2]. D. Kingery (ed.) Learning from Things. Method and Theory of Material Culture Studies, Washington/London: Smithsonian Institution Press, 1996.

[3]. I. Kopytoff, "The Cultural Biography of Thing", in A. Appadurai (ed.) The Social Life of Things. Commodities in Cultural Perspective, Cambridge: Cambridge University Press, 1986, 64-91.

[4]. L. Daston (ed.) Biographies of Scientific Objects, Chicago: Chicago University Press, 2000.

[5]. L. Daston (ed.) Things That Talk: Objects Lessons from Art and Science, New York: Zone, 2004.

[6]. M.G.Santa-Bárbara Contributo para a Recuperação e Integração Museológica do Laboratorio e Amphitheatro de Chimica da Escola Politécnica de Lisboa, Departamento de Antropologia da Faculdade de Ciências Sociais e Humanas. Lisboa: Universidade Nova de Lisboa (Dissertação de Mestrado em Museologia), 2001.

[7]. S. Alberti "Objects and the Museum", ISIS 96 (2001) 559-571.

[8]. S. Lubar, D.W. Kingery (eds.) History from Things. Essays on Material Culture, Washington/London: Smithsonian Institution Press, 1993.

[9]. V. Leitão, M.G.Santa-Bárbara QUIMICA 102(2006) 45-54.

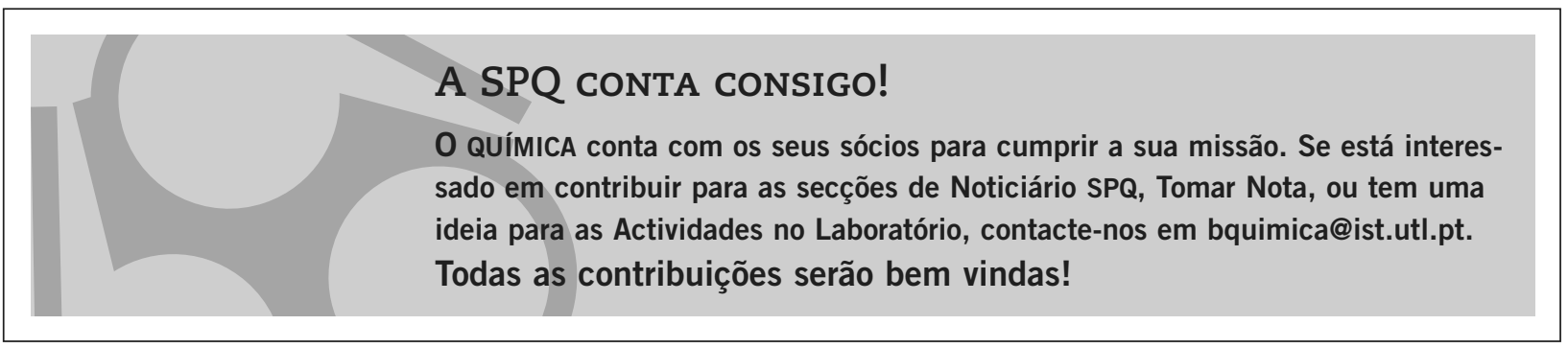

